\title{
Detection of Surface Roughness Evolution of Carbon Steel Subjected to Outdoor Exposure and Constant Humidity Corrosion Tests
}

\author{
Dara To ${ }^{1, *}$, Osamu Umezawa ${ }^{2}$ and Tadashi Shinohara ${ }^{3}$ \\ ${ }_{1}^{1}$ Industrial and Mechanical Engineering Department, Institute of Technology of Cambodia, \\ Russian Conf. Blvd. Phnom Penh, Cambodia \\ ${ }^{2}$ Faculty of Engineering, Yokohama National University, Yokohama 240-8501, Japan \\ ${ }^{3}$ National Institute for Materials Science, Tsukuba 305-0047, Japan
}

\begin{abstract}
The surface roughness evolution of carbon steel under corrosion was studied through outdoor exposure and constant humidity corrosion tests. The statistical analysis of the roughness parameters, such as the degree of skewness and kurtosis, are the good indicators to anticipate the corrosion stages. The results indicated that the corroded surface evolution could be classified into three stages of corrosion. The first stage showed shallow and sparsely distributed pits on the surface of the steel with a probability density function of negative skewness and a high degree of kurtosis $(K u>3)$. The second stage exhibited pits distributed densely and partial overlapping of pits. In the third stage, the overlapping pits entirely covered the metal surface, thereby exhibiting uniform corrosion. Statistical analysis of the corroded surface indicated that the random depth variable of the uniform corrosion follows the normal distribution. The samples exposed to outdoor for one year exhibited the corrosion in the first stage only, while the samples exposed to constant humidity chamber for ten months showed all the three stages of corrosion. [doi:10.2320/matertrans.MF201702]
\end{abstract}

(Received November 27, 2017; Accepted May 18, 2018; Published June 22, 2018)

Keywords: corrosion, surface roughness, probability density function, skewness, kurtosis

\section{Introduction}

Carbon steel has been commonly adopted for various steel structure constructions. Its uniform corrosion, described as a thickness reduction over time, is an expensive problem faced by society. One of the detrimental results of atmospheric corrosion is the collapse of steel structure constructions. Several studies have been performed to investigate the fracture behavior of steel due to corrosion. ${ }^{1-4)}$ The corrosion pit or notch, normally formed in the early stages of corrosion, influences the failure of steel by initiating microcracks, which grow under tensile or cyclic stresses.

Roughness is a reliable indicator of the performance of mechanical structures and components because surface irregularities may present nucleation sites for cracks. $\mathrm{Xu}^{5)}$ estimated the effect of corrosion pits on the fatigue life of steel plates based on a three-dimensional (3D) profile. The study revealed that these pits existed at the early stage of corrosion, and uniform corrosion gradually manifested at the final stage. Wang ${ }^{6}$ ) detected corrosion pits via optical images using gradient-based Hough transform, which detects pits with a variety of radii and depths. Jarrah ${ }^{7)}$ observed corrosion pit interactions by two-dimensional spectral analysis and established it as a powerful technique to distinguish spatial data.

Those suggested methods are effective tools for observing corrosion pits instantaneously. However, detailed study of surface evolution during corrosion is required. Bhushan ${ }^{8)}$ proposed mathematical models and measurement techniques for observing the corroded surfaces. It is suggested that the statistical analysis of the probability distribution of the random depth variable interprets the roughness, especially the skewness and kurtosis which can be applied to pits. Hence,

*Corresponding author, E-mail: itc_darakh@yahoo.com the present study focused on the surface roughness evolution of carbon steel corrosion, where the degree of roughness was evaluated with the skewness and kurtosis.

\section{Experimental Procedure}

\subsection{Corrosion experiments}

The cold-rolled carbon steel sheet (SPCC: Steel Plate Cold-rolled Commercial, $0.12 \mathrm{C}-0.5 \mathrm{Mn}-0.04 \mathrm{P}-0.045 \mathrm{~S}$ in mass $\%$ ) specimens with the thickness of $1 \mathrm{~mm}$ were cut into the size of $150 \times 70 \mathrm{~mm}$, where the longitudinal direction was parallel to the rolling direction. All samples were washed with deionized water, degreased by acetone, and exposure with the inclination of 45 degrees from the horizontal line.

For the atmospheric corrosion test, the samples were exposed to urban (Phnom Penh), and marine (Sihanoukville) atmospheres in Cambodia for one year. A corrosionaccelerated test, i.e. salt spray with constant humidity (SSCH) test, was also performed to characterize the corroded surface after severe corrosion damage, especially effect of long-term atmospheric corrosion attack. The SSCH test was conducted to accelerate the corrosion attack. The sample surfaces were uniformly sprayed by $\mathrm{NaCl}$ solution with a concentration of about $0.045 \mathrm{~mol} / \mathrm{L}$. Thereafter, the samples were kept in a chamber at $25^{\circ} \mathrm{C}$ and a relative humidity of $85 \%$. After the corrosion test, the rust layers were removed by $\mathrm{HCl}$ solution in accordance with ISO/DIS 8403.3.9) Figure 1 represents the sample surfaces before and after removing the corrosion products. After the removal of corrosion products, the surface morphology was observed via a 3D scanner and analyzed using MATLAB.

\subsection{Roughness parameters and method for analysis}

Surface roughness commonly refers to the random deviations in surface height from a mean line, $\mathrm{m}$, or a 

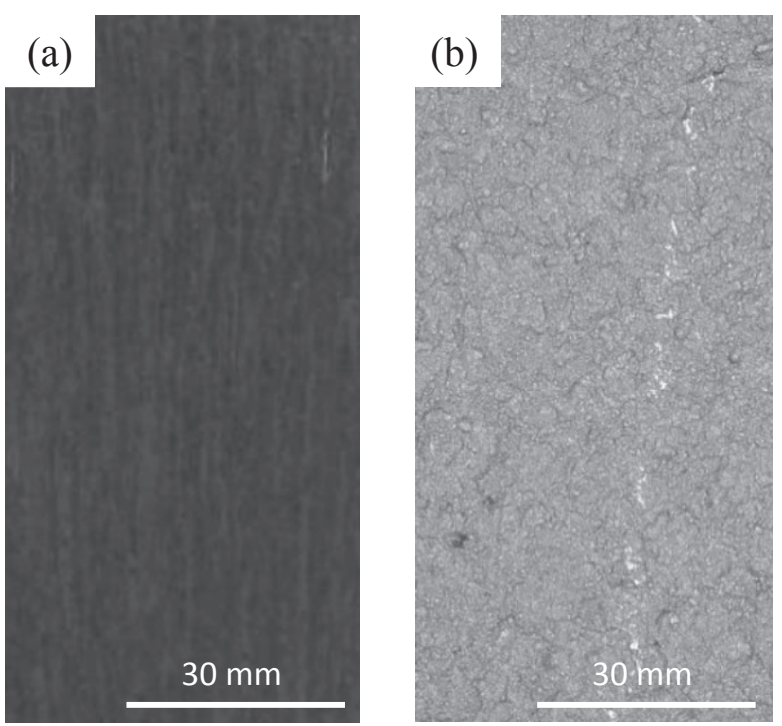

Fig. 1 The sample surfaces before (a) and after (b) removing the corrosion products.

reference plane. It is measured along a single line profile (two-dimensional profile analysis) or along a set of parallel profiles (3D surface profile). In this study, 3D surface profiles were adopted. Maximum height $(R z)$, arithmetic average $(R a)$, standard deviation or variance $(\sigma)$, skewness $(S k)$, and kurtosis $(K u)$ were measured for surface characterization. ${ }^{8)}$ The mathematical expressions of these parameters are listed below:

$$
\begin{aligned}
R_{z} & =\max \mathrm{Z}(\mathrm{x}, \mathrm{y})-\min \mathrm{Z}(\mathrm{x}, \mathrm{y}) \\
R_{a} & =\frac{1}{A} \iint_{A}|Z(x, y)| d x d y \\
\sigma^{2} & =\frac{1}{A} \iint_{A} Z^{2}(x, y) d x d y \\
S k & =\frac{1}{\sigma^{3} A} \iint_{A} Z^{3}(x, y) d x d y \\
K u & =\frac{1}{\sigma^{4} A} \iint_{A} Z^{4}(x, y) d x d y
\end{aligned}
$$

where, $A$ is the sampling area in the $3 \mathrm{D}$ profiles of the metal surface, and $\mathrm{Z}(\mathrm{x}, \mathrm{y})$ is the random depth variable of surface corrosion. Although skewness and kurtosis are rarely used in the statistical analysis of surface roughness, these parameters are pertinent for the characterization of corroded surfaces. Skewness is a measure of the degree of asymmetry of the probability distribution, $\mathrm{P}(\mathrm{z})$. If the left tail is longer than the right, the function exhibits a negative skewness, as illustrated in Fig. 2(a). The reverse indicates positive skewness. When the two tails are equal, it has zero skewness. Skewness is essential to estimate the behavior of corrosion pits or localized corrosions. Kurtosis is a measure of peakedness or flatness of data following a random distribution (Fig. 2(b)). For a smooth face, the data distribution manifests a peak around the mean line or mean plan. In contrast, the data distribution of corroded faces is flat and widely distributed from the mean. Deep corrosion attacks produce a flat distribution with low kurtosis.
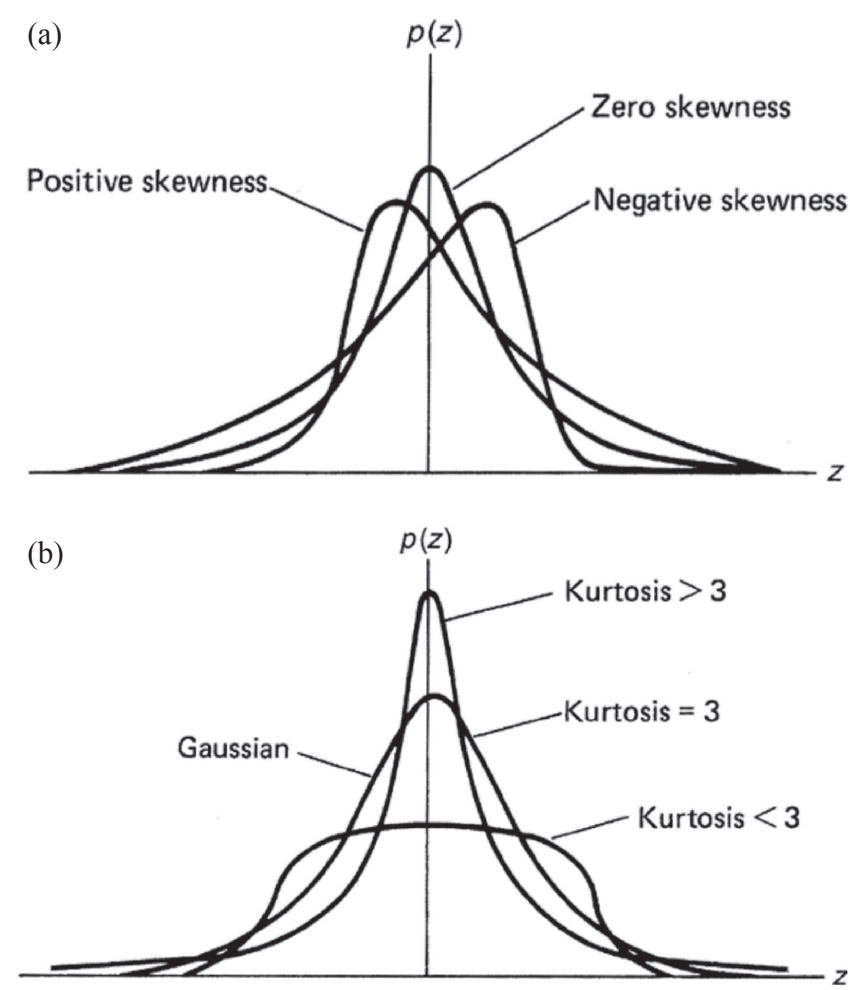

Fig. 2 Probability density functions of a random variable in depth, $\mathrm{Z}(\mathrm{x}, \mathrm{y})$ : (a) skewness and (b) kurtosis. ${ }^{8}$

\section{Results and Discussion}

\subsection{Surface morphology of corroded samples}

Figure 3 shows the surface characteristics of the corroded samples under urban (Phnom Penh) and marine (Sihanoukville) atmospheres in Cambodia for one year exposure test. Base on the weight loss measurement, the corrosion rates under the urban and marine atmospheres were 9 and $27 \mu \mathrm{m} /$ year, respectively. The weight loss was converted to the thickness loss as $70 \mathrm{~g} / \mathrm{m}^{2}$ year $=9 \mu \mathrm{m} /$ year and $210 \mathrm{~g} / \mathrm{m}^{2}$ year $=27 \mu \mathrm{m} /$ year. After the one-year exposure test, numerous pits or notches formed on the surface of the carbon steel samples. The pits are sparsely distributed on the samples under the urban atmosphere, as shown in Fig. 3(a). The pits under marine atmosphere are more concentrated, deeper, and bigger than those under the urban atmosphere (Fig. 3(b)) owing to greater corrosivity in the marine atmosphere than in the urban atmosphere. Interestingly, the atmospheric corrosion tests in both urban and marine atmospheres, showed that the surfaces of the carbon steel samples were not entirely corroded. Instead, a number of pits formed. It is presumed that extending the exposure period will result in complete corrosion of the metal surfaces.

An accelerated corrosion test was adopted to analyze the corroded faces throughout corrosion. Figures 4 and 5 illustrate the corroded surface evaluation during ten-months of SSCH testing. Generally, the corroded surfaces of carbon steel exhibit shallow pits, as their ratios of $a$ with $b$ are above 1 , $(a / b>1)$, as shown in Fig. 4(a). In this study, the corroded surface was classified into three stages of corrosion as follows. In the first stage of corrosion, the shallow pits were sparsely distributed on the metal surface (Fig. 4(a)). 


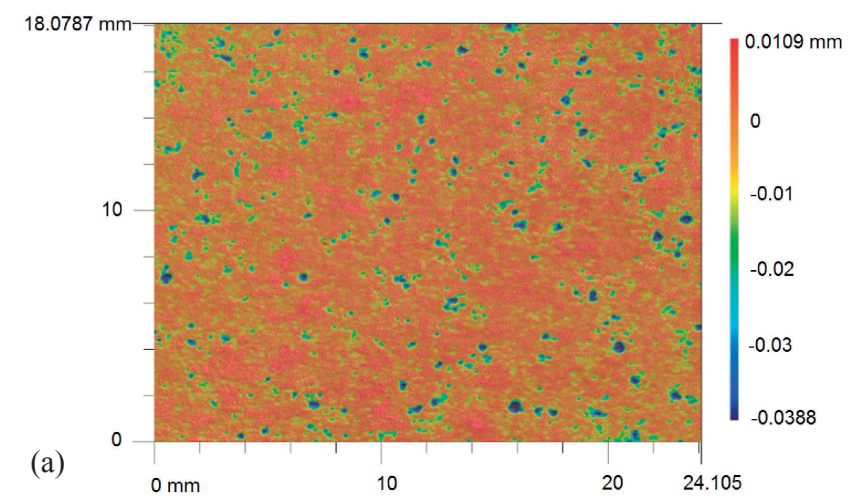

$18.0787 \mathrm{~mm}$

(b)

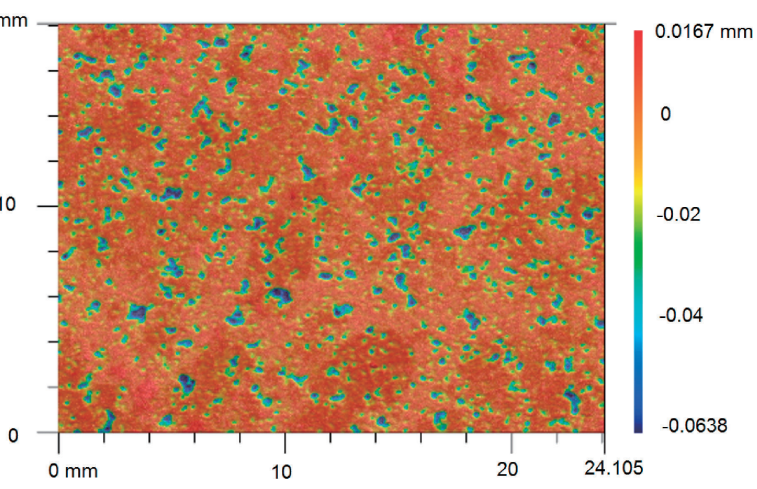

Fig. 3 Surface corrosion of carbon steel under the urban atmosphere (Phnom Penh) (a) and the marine atmosphere (Sihanoukville) (b) in Cambodia for one year.
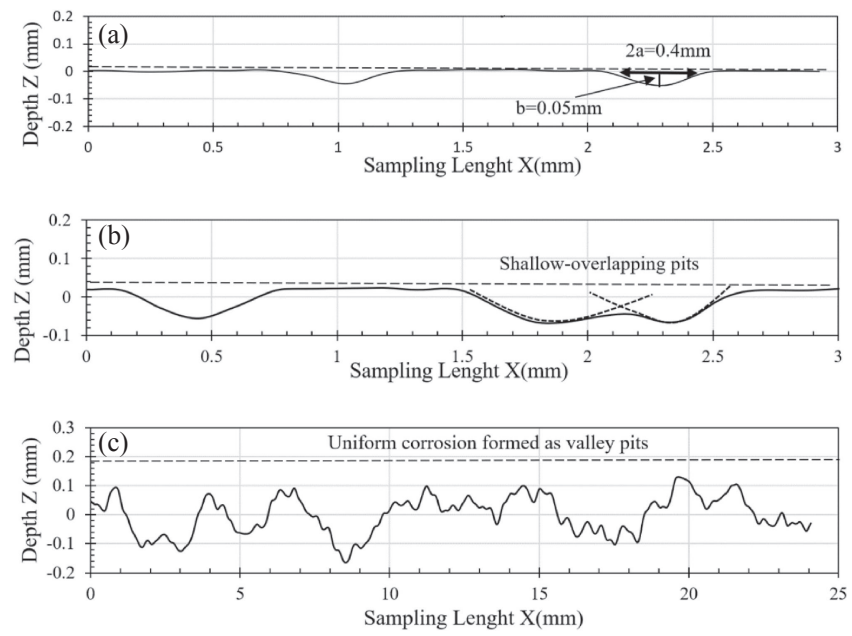

Fig. 4 Two-dimensional profiles of corrosion pit evolution represented in the $(X, Z)$ plan where the $Y$-axis is constant $(Y=0 \mathrm{~mm})$ : (a) shallow pits appeared after six days of SSCH test, (b) overlapping shallow pits appeared after one month of SSCH test, and (c) valley pits formed after ten months of SSCH test (uniform corrosion).

In the second stage, the pit distribution gradually densified, leading to overlapping shallow pits (Fig. 4(b)). After five months of SSCH, the surface of the metal was completely corroded, and the pits transformed into valleys, which was calcified as the third stage of corrosion. This observation, referred to as "uniform corrosion", is shown in Fig. 4(c). Figure 5 shows the morphology of the corroded surface during the ten-month $\mathrm{SSCH}$ test, displaying shallow pits (Fig. 5(a)), overlapping pits (Fig. 5(b)), and valley pits (Figs. 5(c) and 5(d)).
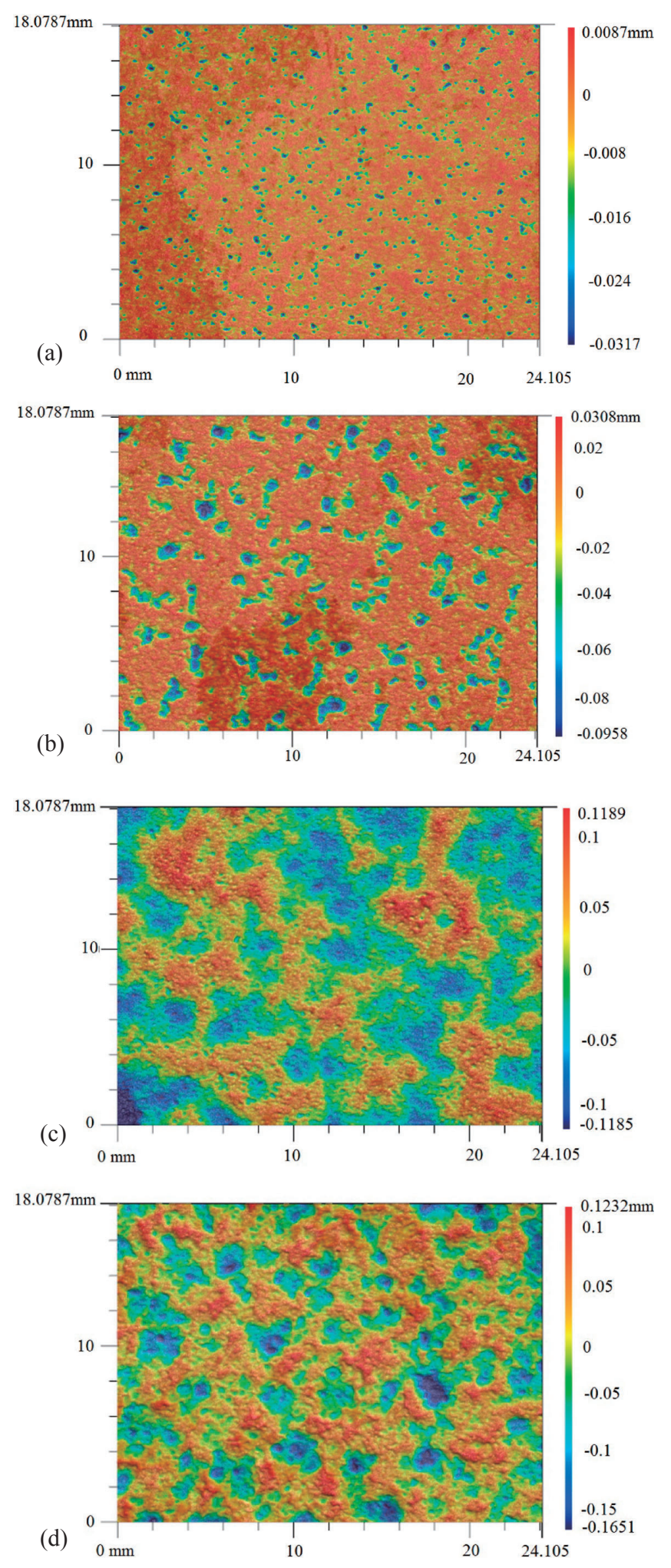

Fig. 5 Surface evolution of carbon steel by SSCH test: (a) six-days (shallow pits), (b) one-month (overlapping shallow pits), (c) five-months (uniform corrosion), and (d) ten-months (uniform corrosion).

The surface morphologies of the samples exposed to outdoor and the SSCH test were comparable. Under one-year of outdoor exposure test, there were many pits formed, and it was very similar to those of six-days SSCH test as seen in the Figs. 3(a) and 5(a). That is because the both cases are in the same stage (first stage) of corrosion. 

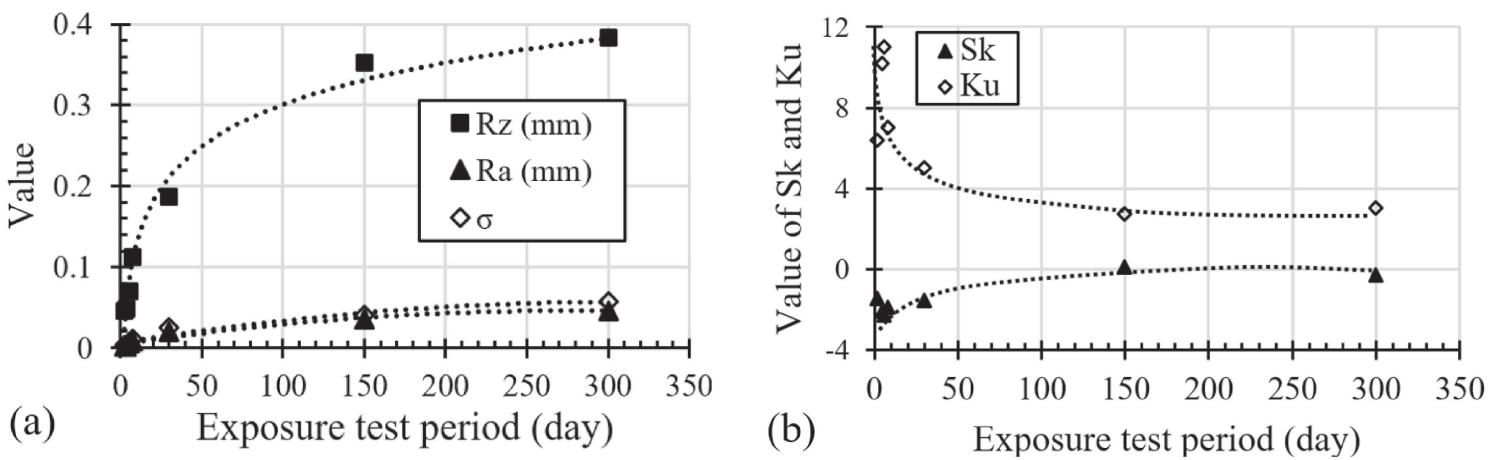

Fig. 6 Evolution of roughness parameters with exposure test time during the SSCH test: (a) evolution of Rz, Ra and $\sigma$, and (b) evolution of skewness $(S k)$ and kurtosis $(K u)$.

\subsection{Roughness parameters}

In the $\mathrm{SSCH}$ test, the degrees of $R z, R a$, and $\sigma$ of carbon steel corrosion were observed to increase with exposure time, as shown in Fig. 6(a). Kurtosis decreased prior to settling at the value of $K u \approx 3$, while the skewness increased prior to settling at the value of $S k \approx 0$, as shown in Fig. 6(b). These phenomena were explained by probability density function (PDF) analysis.

\subsection{Probability distribution of surface corrosion}

Skewness explains the degree of asymmetry from the mean value. For the corroded surfaces, the PDF of the random depth variable, $Z(x, y)$, exhibited negative skewness. This indicates the non-uniform distribution of the random variable $\mathrm{Z}(\mathrm{x}, \mathrm{y})$ due to the presence of pits on the corroded surface (Fig. 7). In this statistical analysis, the 0 value of the $Z(x, y)$ is the mean plan of the corroded surface.

Figure 8 shows the PDF of the corroded samples under marine and urban atmospheres in Cambodia for one-year. In the first month of the atmospheric corrosion test, the variable $\mathrm{Z}(\mathrm{x}, \mathrm{y})$ of the corroded surface was highly concentrated around the mean of zero, corresponding to the sharp peak of the PDF, and was skewed with a long tail to the left, suggesting the initiation of sparse pits. With a prolonged exposure time up to six months and one year, the number of pits increased and their distribution densified. Thus, the

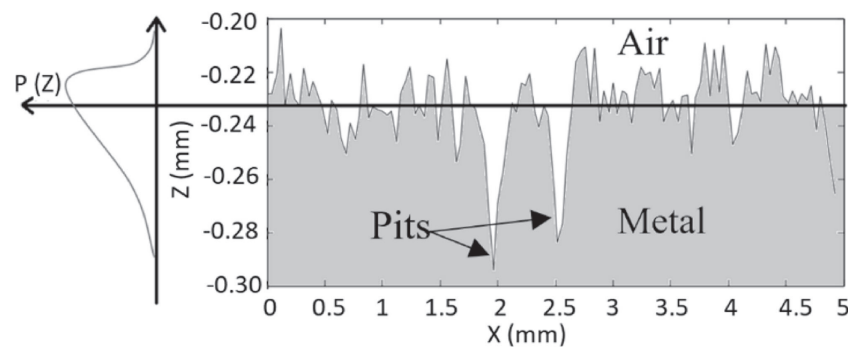

Fig. 7 Probability distribution explains the asymmetry (skewness) of the random variable in high surface corrosion.

PDF of the corroded surface was widely distributed around the mean plane displaying lower peak. Under one-year of outdoor exposure test in both urban and marine atmospheres, the kurtosis value was higher than 3 , indicating that the data distribution was peaked due to the existing of pits on the corroded surface of the samples.

However, the data PDF follows a normal distribution (Figs. 6(b), 9(b) and 9(c)) with degrees of skewness and kurtosis of approximately zero $(S k \approx 0)$ and three $(K u \approx 3)$, respectively, when the carbon steel samples were heavily corroded, as in the five-months of SSCH test. When the data set of corroded surfaces reach a normal distribution, the corrosion is considered as uniform.
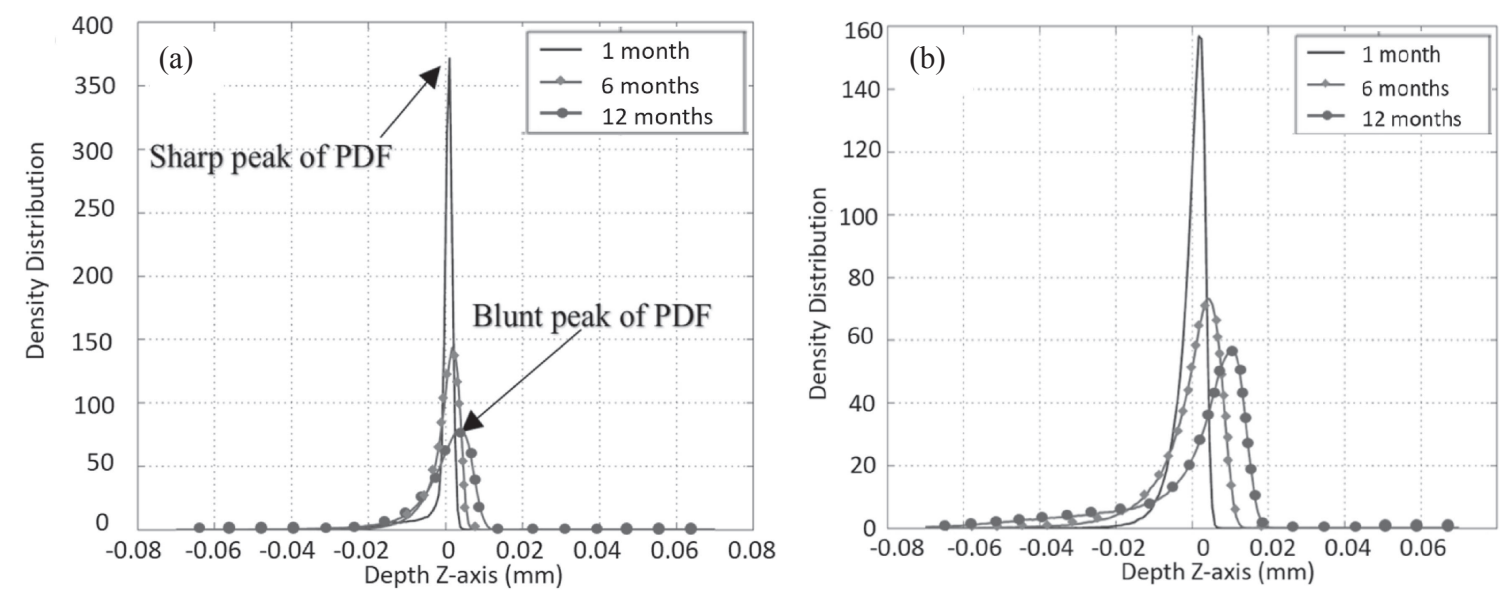

Fig. 8 Probability density distribution of surface corrosion of the samples exposed to urban and marine atmospheres: (a) urban atmosphere and (b) marine atmosphere. 

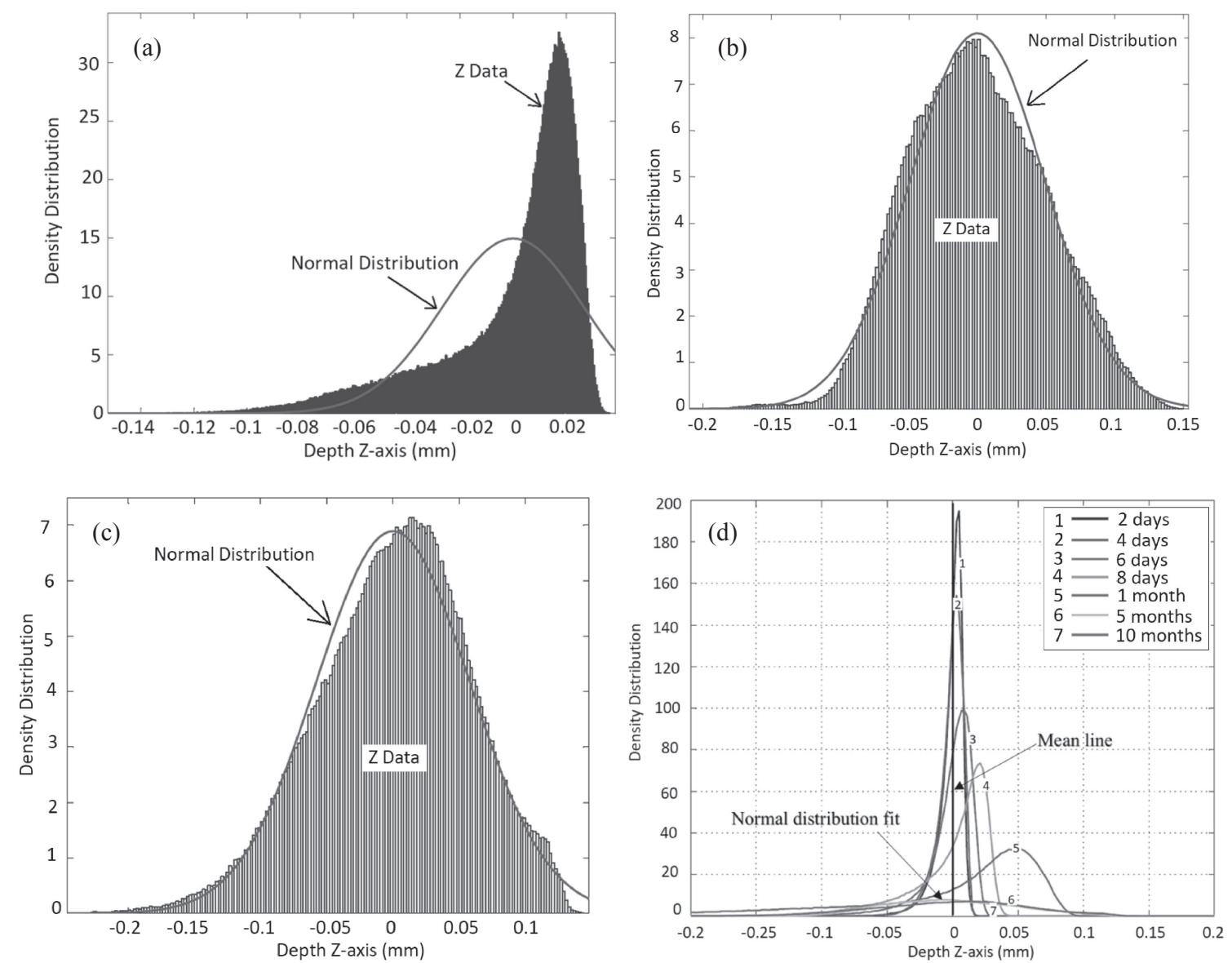

Fig. 9 Probability density distribution of variable $\mathrm{Z}(\mathrm{x}, \mathrm{y})$ on the corroded surface of carbon steel samples subjected to SSCH for ten months: (a) one-month (negative skewness), (b) five-months (normal distribution), (c) ten-months (normal distribution), and (d) PDF evolution.

\section{Conclusions}

The surface roughness of carbon steel under corrosion varies with time and atmosphere types. The maximum height $(S z)$, arithmetic average $(S a)$, and standard deviation $(\sigma)$ increased with exposure time. The corroded surface was classified into three stages of corrosion. In the first stage, the corrosion appeared sparsely; the metal surface exhibited pits or notches distributed with negative skewness and a high degree of kurtosis $(K u>3)$. In the second stage, several pits began to overlap with time; and shallow pits continued to form. In the third stage, the metal surfaces were uniformly covered by rust. Upon complete surface corrosion, the random variable in depth, $\mathrm{Z}(\mathrm{x}, \mathrm{y})$, of the corroded surface followed a normal distribution, with values of $S k \approx 0$ and $K u \approx 3$. According to the $3 \mathrm{D}$ images, the uniformly corroded surface exhibited valleys of shallow pits which insignificantly contribute to fracture mechanism. Thus, the statistical analysis of the roughness parameters, especially the degree of skewness and kurtosis, gives good indicators to anticipate the corrosion stages.

\section{REFERENCES}

1) M.-Q. Li, Z.-W. Wei, F.-S. Zhang and J.-Q. Tang: Corros. Sci. 34 (1993) 1403-1410.

2) B.J. Smith and A.R. Marder: Mater. Charact. 33 (1994) 45-50.

3) T. Chen, J. Nutter, J. Hawk and X. Liu: Corros. Sci. 89 (2014) 146153.

4) S. Ishihara, K. Masuda, T. Namito, S. Sanada and H. Notoya: Int. J. Fatigue 66 (2014) 252-258.

5) S. Xu and Y. Wang: Int. J. Fatigue 72 (2015) 27-41.

6) Y. Wang and G. Cheng: Appl. Surf. Sci. 366 (2016) 9-18.

7) A. Jarrah, J.-M. Nianga, A. Iost, G. Guillemot and D. Najjar: Corros. Sci. 52 (2010) 303-313.

8) B. Bhushan: Surface roughness analysis and measurement techniques, (The Ohio State University, CRC Press LLC, 2001).

9) ISO/DIS 8403.3, Metals and alloys, Procedures for removal of corrosion products from corrosion test specimens, (International Standards Organization, Geneve, 1985). 